\title{
Double and Multiple Star Speckle Observations at the 6-m Telescope
}

\author{
I. I. BALEGA, Y. Y. BALEGA, V. A. VASYUK \\ Special Astrophysical Observatory of the Russian Academy, Zelenchuk \\ region, Karachai-Cherkesia 357147 , Russia \\ J. J. McMANUS \\ Florida Institute of Technology, 150 West University Blvd., \\ Melbourne, FL, USA
}

\section{INTRODUCTION}

During the last 15 years more than 9,000 speckle interferometric measurements of binary stars have been collected using large optical telescopes (McAlister \& Hartkopf 1988). Among them a significant contribution to the world speckle data has been made by the 6-m telscope near Zelenchuk. Up to now this instrument provides the maximal spatial resolution for single-aperture telescopes. First speckle images of the binary Capella were recorded at the telescope in 1977 (Balega \& Tikhonov 1977), but we spent 5 more years to create special television techniques for photon counting and digital means for image processing before we started the regular interferometric program of binary observations in the wide range of stellar magnitudes. At first, the measurements were conducted in cooperation with French astronomers from the Centre d'Etudes et de Recherches Geodynamiques et Astronomiques using the optical camera and the television detector developed by Blazit et al. (1977). Since 1983 our equipment has been in use. The program of observations was oriented upon the traditional problems of multiple star speckle interferometry:

1. Determination of stellar distances and masses for different types of binaries whose orbital elements can be derived. This includes already known fast visual and astrometric pairs with undetermined orbits, spectroscopic binaries that can be resolved directly, and newly discovered interferometric pairs which show fast orbital motion. The main attention was devoted to the late-type dwarfs in the vicinity of the Sun.

2. Search for the secondary components whose existence could explain anomalies of stellar spectra or photometry (stars with composite spectra, occultation binaries, etc. )

3. Study of unusual binaries (symbiotic stars, binaries with relativistic components, such as SS 433 , etc. )

\section{TECHNIQUES}

Measurements were made in the prime $\mathrm{f} / 4$ focus of the telescope. We used a photon counting television camera built on the base of the SIT tube and microchannel plate image intensifier. The frame frequency of the camera is $\mathbf{5 0}$ 
$\mathrm{Hz}$ with the image format $256 \times 256$ pixels. Special centroiding electronics gives coordinates of the photon events in real time. The camera is sensitive in the spectral range $200-800 \mathrm{~nm}$. For the most part, the $600 / 14 \mathrm{~nm}$ interference filter was used during observations. For some binary stars we also applied $650 / 20 \mathrm{~nm}$ and $510 / 10 \mathrm{~nm}$ filters. Usually we were not able to shift to shorter than $500 \mathrm{~nm}$ wavelength because of seeing limitations. The shutter has been used to select short exposures between 5 and $20 \mathrm{~ms}$.

Using a pair of microscope objectives the magnification of the optical camera has been optimized to give a pixel size, in the plane of photocathode, equal to $0 " .012$ or $0^{\prime \prime} .006$. Usually smaller magnification has been used for measurements of fainter stars. We used a graticule, finely devided in two dimensions with a step of 10 microns, for the image scale calibration and for the correction of television field distortions. The graticule has been installed in front of the microobjective. The final scale has been established for each run of observations using the focus value of the 6 - $\mathrm{m}$ mirror, $f=24025.5 \pm 8 \mathrm{~mm}$, found in the course of its certification in the factory.

For bright objects, $\mathrm{V}<8$ magnitude, a two-dimensional autocorrelation function of speckle images has been computed in real time with the help of a hard-wired image correlator and integrated in computer memory. The process of the correlation acquisition was displayed on the monitor. For fainter stars a time-resolved imaging mode has been used, allowing to store the photon event coordinates on magnetic tapes. In this case the image autocorrelation can be found with the help of the computer after the cleaning and image selection procedure. During the observations we have usually begun from the estimation of the central speckle peak intensity in the autocorrelation. With typical conditions with a $10^{\text {th }}$ magnitude star our camera recorded about 2000 photons per second. To obtain a well-defined secondary peak in the autocorrelation of a double star, about 10 photon events had to be processed, we required 5-10 min of integration.

\section{STATISTICS OF SPECKLE OBSERVATIONS}

By the end of 1990 about 600 measuremens of 253 binary, and suspected binary stars were obtained through this program using the speckle interferometer at the 6-m telescope near Zelenchuk. The majority of these observations were collected in a separate catalogue, with the most recent data from December 1988 (Balega et al. 1990). The speckle data given in the catalogue present only $7 \%$ of the world's speckle material compiled in the Second Catalogue of Interferometric Measurements of Binary Stars (McAlister \& Hartkopf 1988). It should be noted that the 6-m telescope has been used mainly for study of compact pairs because they benefit most from the speckle interferometry with highest resolution. The histogram of angular distances for our measures in comparison with the material collected in the Second Catalogue is given in Figure 1 (note that the Second Catalogue includes the data obtained in Zelenchuk). Distributions of our measures for different spectral classes and stellar magnitudes are shown in Figures 2 and 3. A large majority of stars included in the program are bright objects, therefore they already have information (visual, interferometric, photometric, spectroscopic, or astrometric) concerning their binary nature. Although most 


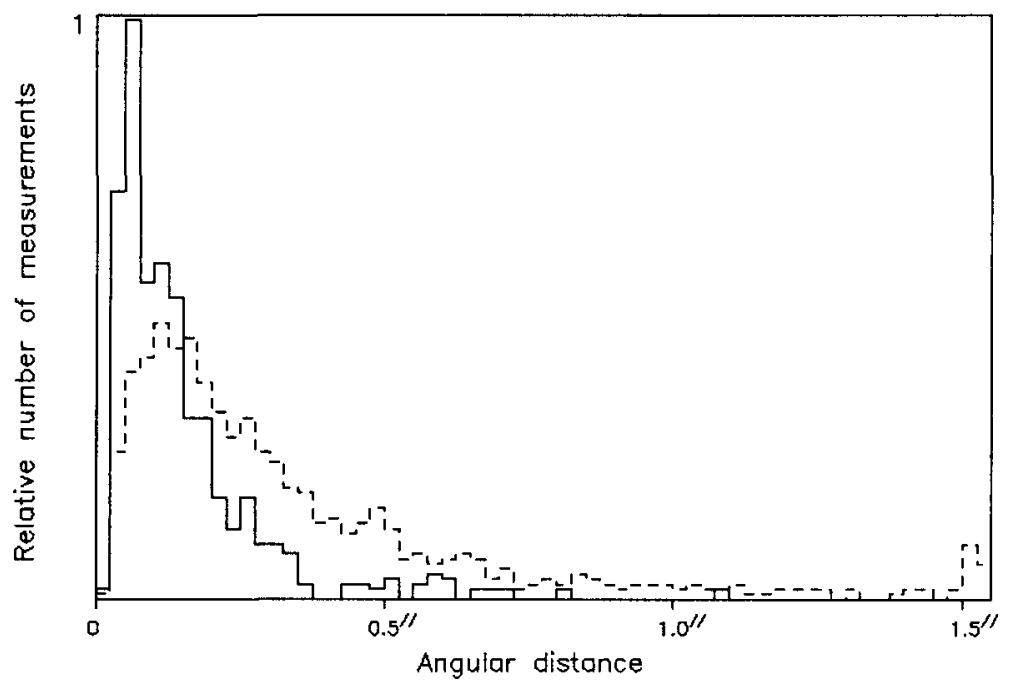

FIGURE 1. The distribution of the measured angular separations for the speckle data collected in the Second Cataloque of McAlister \& Hartkopf (1988) - dashed line, in comparison with the speckle observations from Zelenchuk - solid line. The areas of the two histograms are normalized.

of the observed pairs are bright, the photon counting speckle camera has been shown to be capable of observing stars as faint as $\mathrm{V}=15$.

In 147 cases the observed stars were not resolved, this can be explained by one, or a combination of the following reasons:

1. distance between the components was less than 0 ".02-0".03;

2. brightness difference between the components exceeded an atmospherically defined limit, usually 3 magnitudes;

3. atmospheric turbulence was too fast for effective speckle registration.

A mean-squared error of a single measurement for the catalog is close to $0 \prime .003$ for the position angle $\theta$, as well as for the angular distance $\rho$. The error was established through repeated recording of autocorrelations for the stars taken as standards. The accuracy is higher for bright stars, $s=0 \prime .0015$, and falls to $s$ $=0^{\prime \prime} .005$ for the objects fainter than $V=8$. The position angle error becomes significant for very close pairs. In case of a binary separated by 0 ".03-0".05 it can reach 10 even for zero magnitude difference between the components.

In many cases the pairs with an angular separation close to the telescope's diffraction limit were resolved: HR $233=$ McA 2, $\mu$ Ari, 66 Ari, 36 Tau $=$ McA 13, $65 \mathrm{Gem}=\mathrm{McA} 31 \mathrm{Aa}, 19 \mathrm{Leo}=\mathrm{McA} 34,4 \mathrm{UMi}$, and others. The widest binary observed in the program was Kui 79 with $1^{\prime \prime} .1$ distance between the components. The upper limit is defined by the digital window of the correlator. A $128 \times 128 \times 16$-bit autocorrelogram memory in conjunction with one 


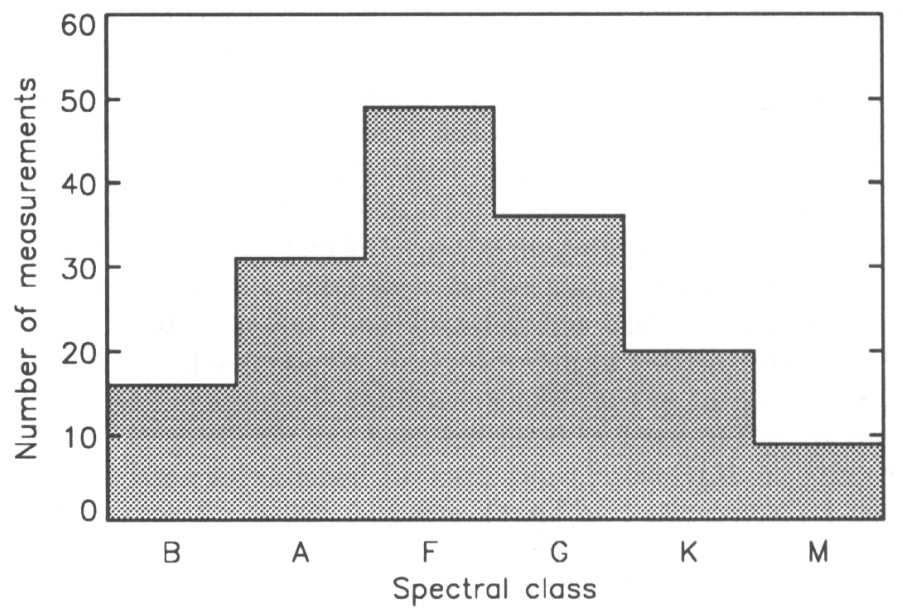

FIGURE 2. The distribution of spectral classes for the binaries measured at the 6-m telescope.

from among two microscope objectives provides angular windows of 0 " $8 \times 0$ " .8 and $1^{\prime \prime} .6 \times 1^{\prime \prime} .6$, respectively for high and low magnification. Seven systems were directly resolved for the first time: 6 Per $\left(\rho=0^{\prime \prime} .040\right)$, 66 Ari $\left(\rho=0^{\prime \prime} .033\right)$, $+19^{\circ} 0662\left(\rho=0^{\prime \prime} .066\right),+23^{\circ} 1346\left(\rho=0^{\prime \prime} .105\right), 4$ UMi $\left(\rho=0^{\prime \prime} .020\right),+112874=$ Gliese $600(\rho=0 " .077)$, and ADS 14864 Aa $\left(\rho=0^{\prime \prime} .096\right)$. The angular distances are given for the epochs of the first resolution.

Using our speckle data and the results from the interferometric program at Kitt Peak, we have computed new orbits for 8 binaries with periods in the range $1.5-13$ years. Many previously known orbits were improved on the base of recent observations at the 6-m telescope. A few of them give an idea about their compactness, and are presented in Figure 4 together with their orbital elements. $\mathrm{n}$ To show satisfactory agreement between our speckle data and the data from Kitt Peak we can analyze the example of the close pair HR 233 with a period of 12 years. To compute the orbit all 13 interferometric measures published before 1987 were used with equal weights. The angular distance $\rho$ between the components of this pair never exceeds $0^{\prime \prime} .05$, therefore only the largest telescopes make these measurements possible. The mean-squared errors for the 6 observations on Kitt Peak are $s=0 " .0048, s=0 \prime .0078$, while for the 7 observations in Zelenchuk $s=0^{\prime \prime} .0023, s=0^{\prime \prime} .0024$. We believe that in this case smaller residuals for the data from the 6-m telescope show the advantages of the larger aperture, when binaries at the limit of resolution are observed. In the case of 51 Tau, whose orbit is defined with better accuracy because of larger angular dimensions, we find similar residuals for the two instruments: $s=0^{\prime \prime} .0038, \mathrm{~s}=$ $0 " .0065$, and $\mathrm{s}=0.0038, \mathrm{~s}=0.0052$, for the measures made at $4-\mathrm{m} \mathrm{KPNO}$ and 6-m SAO, respectively. We conclude from these data that there is no significant systematic difference in the results from Kitt Peak and Zelenchuk. 


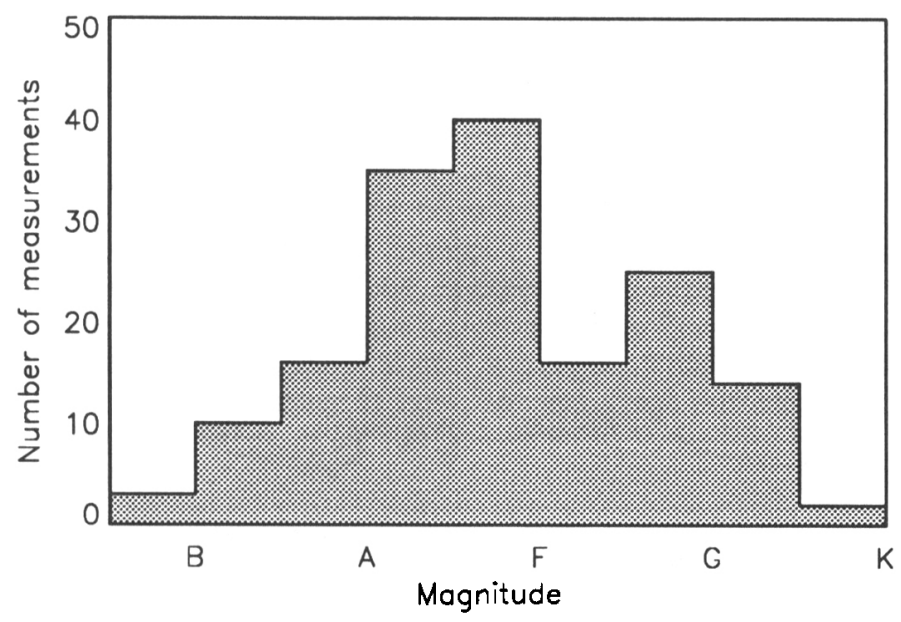

FIGURE 3. The distribution of stellar magnitudes from the catalogue of speckle measures at the 6-m telescope.

It is interesting here to note an effect of overestimating angular distances between the components existing in speckle interferometry in the cases when very close binaries, near the diffraction limit, are observed. Similar systematic differences have been found by Tokovinin (1988) between interferometric measurements with large telescopes and visual observations of close pairs with micrometers. Usually visual observers give values of $\rho$ that are $20-40 \%$ higher than the ones found with a speckle interferometer and larger aperture. Another interesting but still poorly explained effect, often present in our speckle observations of close pairs, is a distortion of the central speckle peak of autocorrelation that could be assumed to be an indication of a secondary companion. This distortion has an evident azimuth effect, showing the influence of the air mass transfer inside the dome and in the nearby surrounding area.

In addition to more or less systematic speckle observations of binaries, a few special observational programs were organized at the telescope. First, we have made an attempt to find binaries between carbon giant stars. Up to this moment, masses and luminosities of these stars remain undetermined. This can be explained in part by the fact that among carbon stars we still have not found binary systems showing orbital motion. During the years 1984-1986, 38 carbon stars were analyzed by means of the speckle camera at the telescope. The secondary companion, at $\theta=123^{\circ}, \rho=0^{\prime \prime} .12$ with $\Delta \mathrm{m}=2.5$, was suspected only for one of them - UX Dra (Alksnis et al. 1988). Beyond a doubt, failure to find more carbon pairs can be partly explained by the usage of only $600 \mathrm{~nm}$ and 510 $\mathrm{nm}$ filters. Unfortunately, because of the atmospheric situation we could not apply a blue filter where the probability of detection of a hypothetical component would be considerably higher. Second, speckle interferometric observations of dwarfs from the Gliese catalog of nearby stars, showing variable radial velocity, 

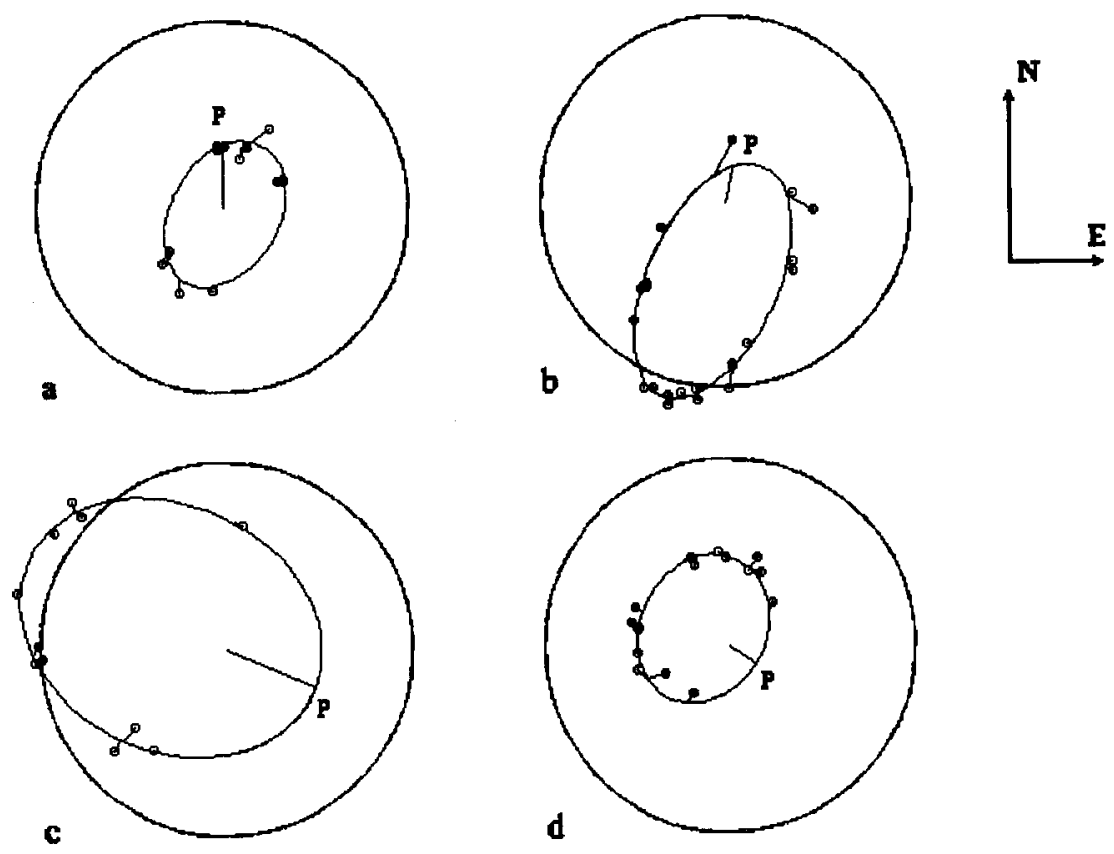

FIGURE 4. Speckle interferometric orbits for 4 binaries derived from the measures obtained mainly at the KPNO 4-m (open circles) and the SAO 6-m (filled circles) telescope. All the orbits are plotted to the same scale with the main stars in the centres of circles of $0^{\prime \prime} .1$ radius.

(a) $\mathrm{HR} 233=\mathrm{McA} 2$

$\left(\mathrm{T}=1984.12, \mathrm{P}=12.17 \mathrm{y}, \mathrm{e}=0.084, \mathrm{a}=0^{\prime \prime} .042, \mathrm{i}=134^{\circ}, \Omega=150^{\circ}, \omega=140^{\circ}\right)$

(b) HR $6469=$ McA 47

$\left(\mathrm{T}=1980.835, \mathrm{P}=5.504\right.$ y, $\left.\mathrm{e}=0.679, \mathrm{a}=0^{\prime \prime} .074, \mathrm{i}=53^{\circ} .7, \Omega=141^{\circ} .7, \omega=221^{\circ} .1\right)$

(c) $+58^{\circ} 1929=\mathrm{Gl} 762.1=\mathrm{McA} 56$

$\left(\mathrm{T}=1981.205, \mathrm{P}=1.355\right.$ y, $\left.e=0.397, \mathrm{a}=0^{\prime \prime} .084, \mathrm{i}=147^{\circ} .9, \Omega=61^{\circ} .1, \omega=172^{\circ} .4\right)$

(d) $\mathrm{l} \mathrm{Cyg}=\mathrm{ADS} 14296 \mathrm{Aa}=\mathrm{McA} 63 \mathrm{Aa}$

$\left(\mathrm{T}=1982.19, \mathrm{P}=11.63 \mathrm{y}, \mathrm{e}=0.524, \mathrm{a}=0^{\prime \prime} .048, \mathrm{i}=135^{\circ}, \Omega=150^{\circ}, \omega=272^{\circ}\right)$

were carried out in hope of their direct resolution. This program was initiated by A. Tokovinin from Moscow University to fill a gap in the lists of resolved binaries among low-mass stars with short periods. The program included only small velocity amplitude spectroscopic pairs. As a result, of the 21 stars observed, only Gliese $600=+11^{\circ} 2874$ was resolved for the first time (Balega et al. 1991). Finally, in cooperation with Estonian Academy of Sciences, speckle data were collected during 1983-1986 for the symbiotic star CH Cyg in order to compare its high-resolution optical structure with radio maps and to detect the white dwarf companion. This program also led to a large collection of negative results. 


\section{NEW POSSIBILITIES}

New technical possibilities have opened up in the field of binary star interferometry during the last few years. Due to the introduction of the time-resolved photon counting mode we can provide speckle photometry of binaries, as well as complete image restoration in the presence of noise with the help of bispectral analysis. This technique has already been applied for the restoration of images of triple stars, such as ADS 11344 (Balega et al. 1988). Sub-diffraction limited angular resolution, up to $0^{\prime \prime} .002-00^{\prime \prime} .004$, is expected as a result of the introduction of differential speckle imaging at the telescope. The first of these experiments held in the observatory for the classical binary Capella, in cooperation with astronomers from the University of Nice, showed great potential in the field of binary star research (Petrov et al. 1991).

\section{CONCLUSION}

The 6-m telescope near Zelenchuk can effectively be used for the regular speckle astrometry of binary stars. Binary observations with the television speckle camera and digital procedures for reducing speckle data provide a routine precision of $0^{\prime \prime} .003$ for the angular distances, as well as for the position angles. The observations can be held in a wide range of stellar magnitudes. What is badly needed now, is a co-ordination of speckle programs at different telescopes. The reason for this lies in the difficulties obtaining observing time, at world largest telescopes, for binary star astrometry. Therefore it is important to begin coordinating the efforts of the interferometric groups from different observatories in order to obtain data for the highest possible number of interesting pairs.

\section{REFERENCES}

Alksnis, A., Balega, I.I., Balega, Y.Y., Doontsans, L., \& Schmeld, I. 1988, Invest. of the Sun and Red Stars, (Riga, Publ. Hause Zinatne), 29, 22

Balega, I.I., Balega, Y.Y., \& Vasyuk, V.A. 1990, Commun. of the Special Astrophys. Obs., 65, Stavropol, 5

Balega, I.I., Balega, Y.Y., Vasyuk, V.A., \& Tokovinin, A.A. 1991, Soviet Astron. Lett., 17,530

Balega, Y.Y., and Tikhonov, N.A. 1977, Soviet Astron. Lett., 3, 197

Balega, Y.Y., Vasyuk, V.A., \& Orlov, V.G. 1988, Commun. of the Special Astrophys. Obs., 59, Stavropol, 74

Blasit, A., Bonneau, D., Koechlin, L., \& Labeyrie, A. 1977, ApJ, 214, L79

McAlister, H.A., and Hartkopf, W.I. 1988, Second Catalogue of Interferometric Measurements of Binary Stars, CHARA Contrib. No.2, (Atlanta, Georgia State Univ.)

Petrov, R., Balega, Y., Blazit, A., Borgnino, J., Foy, R., Lagarde, S., Martin, F., \& Vassyuk, V. 1991, in High-Resolution Imaging by Interferometry, J.Beckers and F.Merkle, eds., (Garching, FRG), ESO Conf. Proc., in press

Tokovinin, A.A. 1983, Soviet Astron. Lett., 9, 627 


\section{DISCUSSION}

GRIFFIN: I think it's not quite fair to illustrate the relative accuracies of observations made with different telescopes by the residuals from your orbit of HR 233. You've got entirely the wrong orbit the HR 233! You have a period of $12.17 \mathrm{yr}$, but the true period is $5.72 \mathrm{yr}$.

BALEGA: The orbit of HR 233 was computed 5 years ago. At that time only 13 measurements of the binary were available. I agree that it is better to use 51 Tauri, with its very well defined orbit, for comparison of relative accuracies of speckle data from different telescopes.

MATTEI: For your observations of carbon stars, may I ask what criteria you use in selecting what to observe?

BALEGA: The list was compiled using already existing information (photometric and spectroscopic) about possible binary nature of these stars.

SINACHOPOULOS: Don't you lose accuracy of position angle determination due to the alt/az mounting of the $6-\mathrm{m}$ telescope?

BALEGA: Because of the azimuthal mounting of the telescope, we use only short, 1 or 2 minutes, integrations of correlations. The speckle camera always has a vertical orientation. To find the north direction, we compute the parallactic angle for each integration.

KAROVSKA: The errors in the positions are impressive. However, I would call for a caution in particular in view of the possible errors introduced by the residual dispersion, instrument distortions, and changes in the telescope focus.

BALEGA: I think that only instrumental distortion is really a big source of uncertainty in position determination. We try to correct it using a graticule that can be installed in front of the microscope objective.

McALISTER: The image tube distortion of concern here is really only a second order effect because we are making differential measurements across small portions of the intensifier tube (which is the source of this distortion). While the maximum distortion from center to edge might be a few percent, or even larger, the effect on measuring the separations of adjacent close speckles averaged over the field is smaller. A proper calibration for scale, for example using a double-slit aperture mask, also tends to compensate for such distortions.

QUIRRENBACH: The accuracy of speckle measurements can be checked by Michelson interferometry, since these methods overlap in the range of 30 to 150 mas. Specifically, the bias in separation close to the resolution limit of speckle observations can be addressed this way. In the Mark III group, we have recognized the need for this comparison, and we will devote some observing time to this effort. 